\title{
Rocky Mountain Labs: NIAID's Montana campus
}

$T$ he Division of Intramural Research (DIR) is a branch of the National Institute of Allergy and Infectious Diseases (NIAID). A fact not widely known about the DIR is that more than $20 \%$ of its research is conducted in western Montana at the Rocky Mountain Laboratories (RML) (Figure 1). Furthermore, RML soon will house one of the very few biosafety level four (BSL4) facilities - laboratories with the strictest levels of biosafety, biocontainment, and security - in the US.

The DIR conducts basic, translational, and clinical research related to immunology, allergy, and infectious diseases, with the aim of promoting the development of new vaccines, therapeutics, and diagnostics to improve human health. At RML, the specific research focus is infectious microorganisms that cause disease in humans and animals. This focus reflects the history of RML, whose most well-known alumni are probably Herald Rea Cox and Gordon Davis, who were involved in identifying Coxiella burnetii, the vector-borne bacterium that causes Q fever, and Willy Burgdorfer, who isolated Borrelia burgdorferi, the vectorborne spirochete that causes Lyme disease.

Even before the first RML building was completed in 1928, researchers were working in the area (in makeshift cabins and tents) to determine the cause of Rocky Mountain spotted fever, a disease that in the early 1900s was lethal in nearly four out of every five cases and that is still sometimes lethal today. Among those who

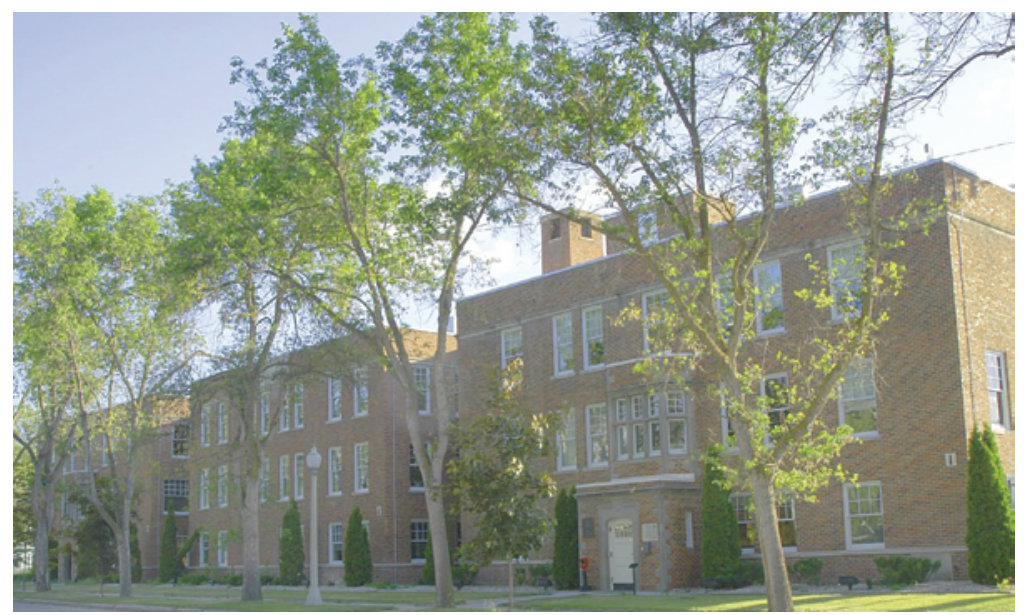

Figure 1

The 33-acre RML campus is located in Hamilton, Montana, a small community between the Bitterroot and Sapphire Mountains. Image credit: NIAID/RML. traveled to work in the area each summer was Howard Ricketts, from the University of Chicago, who in 1909 isolated Rickettsia rickettsii as the Gram-negative bacterium that causes Rocky Mountain spotted fever. Although research into vector-borne diseases continues at RML, there are also programs investigating numerous other infectious diseases. Indeed, RML houses five of the twenty-one laboratories (which Marshall Bloom, associate director of RML, told the JCI can be thought of as departments within a university) that make up the NIAID DIR - the Laboratory of Human Bacterial Pathogenesis, the Laboratory of Intracellular Parasites, the Laboratory of Persistent Viral Diseases, the Laboratory of Zoonotic Pathogens, and the newly created Laboratory of Virology. In addition, the RML has a Veterinary Branch that provides crucial support to the researchers and houses two central facilities used by researchers at all NIAID sites - the Genomics Unit and the Microscopy Unit.

Both Bloom and Kathy Zoon, director of the DIR and scientific director of the NIAID, emphasized to the JCI that, in their opinion, the presence of these central facilities and the Veterinary Branch is one of the most important features of RML, as these divisions allow the researchers to focus on their work. In addition, Bloom said that locating the central facilities at RML promotes interaction with researchers at different sites, in particular those at the main campus in Bethesda, Maryland. He was particularly enthusiastic about the future impact of the new microscope that the Microscopy Unit will house, which is the most advanced of its kind and will be only the second one operating in the entire NIH. This advanced technology will perform cryoelectron tomography, allowing researchers to visualize cells at EM resolution but in three dimensions and their nearly native state.

Bloom said that new technology has rendered the distance between Bethesda and RML almost immaterial. This was echoed by Anthony Fauci, director of the NIAID, who told the JCI that the electronic age has made it seem as if RML is just across the street from the Bethesda campus. RML researchers have video-conferencing facilities freely available and are able to participate interactively in many seminars hosted by the DIR at the Bethesda campus. In fact, Bloom noted that working at RML allows one to perform cutting-edge NIAID research while living in a small community in a beautiful rural setting. He went on to say that this was an opportunity granted to few and one that most RML employees really appreciate. He also believes that the great work-life balance that can be achieved at RML is helping them recruit world-class researchers at all levels.

The most recently recruited laboratory chief at RML, Heinz Feldmann, who is chief of the Laboratory of Virology, will also serve as chief scientist of the newly built BSL4 laboratories. These BSL4 laboratories, which are the first to be operated by the NIAID DIR, are in a brand new building that also houses BSL2 and BSL3 laboratories as well as administrative offices and conference rooms. The BSL2 laboratories have been in use since spring 2008, and Bloom says that the BSL4 laboratories will be ready for use once the CDC completes the final stages of certification, validation, and inspection, something that Bloom hopes will occur in the next couple of months.

Fauci summed up the feeling about RML at the NIAID by telling the JCI that over the last 20-25 years, the potential liabilities of the physical separation between RML and Bethesda have morphed into assets, where the beauty of western Montana and the collegial working environment couple with state-of-the-art facilities to make RML highly attractive to world-class researchers and an integral part of the DIR.

\section{Karen Honey}

